\title{
Existence Results for a Second Order Impulsive Neutral Functional Integrodifferential Inclusions in Banach Spaces with Infinite Delay
}

\author{
V. Kavitha, M. Mallika Arjunan*, C. Ravichandran \\ Department of Mathematics, Karunya University, Karunya Nagar, Coimbatore-641 114, Tamil Nadu, India. \\ Dedicated to George A Anastassiou on the occasion of his sixtieth birthday \\ Communicated by Professor G. Sadeghi
}

\begin{abstract}
A fixed point theorem for condensing maps due to Martelli combined with theories of a strongly continuous cosine family of bounded linear operators is used to investigate the existence of solutions to second order impulsive neutral functional integrodifferential inclusions with infinite delay in Banach spaces.
\end{abstract}

Keywords: Second order impulsive integrodifferential inclusion, cosine functions of operators, mild solution, Martelli's fixed point theorem.

2010 MSC: Primary 34K30, 34K45, 34A60; Secondary 47D06.

\section{Introduction}

The impulsive differential equations have received much attention during the last decade, but the study of the impulsive differential inclusions is relatively late in the literature. The dynamical systems, which involve the jumps or discontinuities are modeled on the impulsive differential equations and inclusions. On the other hand, integrodifferential equations are encountered in many areas of science, where it is necessary to take into account aftereffect or delay (for example, in control theory, biology, ecology and medicine). Especially, one always describes a model which possesses hereditary properties by integrodifferential equations in practice. The theory of integrodifferential inclusions with impulse actions has not yet been fully investigated, when compared to that of impulsive differential inclusions and integrodifferential inclusions. For more details on impulsive theory and integrodifferential equations we refer to the monographs of Bainov and Simeonov [3], Lakshmikantham, Bainov, and Simeonov [43, Samoilenko and Perestyuk [51, Benchohra, Henderson and Ntouyas [7] and the papers of Rogovchenko [54, Liu [47, Hernandez [28, 29, 30, 31, 32, 33,

\footnotetext{
* Corresponding author

Email addresses: kavi_velubagyam@yahoo.co.in (V. Kavitha), arjunphd07@yahoo.co.in (M. Mallika Arjunan), ravibirthday@gmail.com (C. Ravichandran)
} 
Anguraj et al. [2, Balachandran et al. [4, 5, 50, Benchohra et al. [8, 9, 10, Ntouyas [49, Chang et al. [15, 16, 17, 18, 19, Liang et al. 45. However, very few results are available for impulsive differential and integrodifferential inclusions; see for instance, the papers of Benchohra et al. [11, 12, 13, 14, Erbe and Krawcewicz [22, and Frigon et al. 23], Xianlong Fu et al. 24, Anguraj et al. 20] and Junhao $\mathrm{Hu}$ et al. 39].

Abstract neutral differential equations arise in many areas of applied mathematics. For this reason, they have largely been studied during the last few decades. The literature related to ordinary neutral differential equations is very extensive, thus, we refer the reader to 26 only, which contains a comprehensive description of such equations. Similarly, for more on partial neutral functional differential equations and related issues we refer to Adimy and Ezzinbi [1], Hale [27, Wu and Xia [55] and [56] for finite delay equations, and Hernandez and Henriquez [34, 35] and Hernandez [36] for unbounded delays.

Recently, in many areas of science there has been an increasing interest in the investigation of functional differential equations incorporating memory or aftereffect, i.e., there is the effect of infinite delay on state equations. We refer the reader to Kolmanovskii and Myshkis [41, 42, Wu [55] and references therein for a wealth of reference materials on the subject. Therefore, there is a real need to discuss functional differential systems with infinite delay. And the development of the theory of functional differential equations with infinite delays depends on a choice of a phase space. In fact, various phase spaces have been considered and each different phase space has required a separate development of the theory (Hino et al. [37]). The common space is the phase space $\mathcal{B}$ proposed by Hale and Kato [25, which is widely applied in functional differential equations with infinite delay and references therein. However, in this paper, we introduce an abstract phase space $\mathcal{B}_{h}$ which has been adopted by [15, 18, 57. Based on the phase space $\mathcal{B}_{h}$, Chang et al. 15] proved the existence of solutions of impulsive partial neutral functional differential equations with infinite delay:

$$
\begin{aligned}
\frac{d}{d t}\left[x(t)-g\left(t, x_{t}\right)\right] & =A x(t)+f\left(t, x_{t}\right), t \in J=[0, b], \quad t \neq t_{k}, \quad k=1,2, \ldots, m \\
\left.\Delta x\right|_{t=t_{k}} & =I_{k}\left(x\left(t_{k}^{-}\right)\right), \quad k=1,2, \ldots, m \\
x(t) & =\varphi \in \mathcal{B}_{h} .
\end{aligned}
$$

To the best of our knowledge, there is no work reported on a second order impulsive partial neutral functional integro-differential equations and inclusions with infinite delay $\mathcal{B}_{h}$. To close the gap, motivated by the above works, the purpose of this paper is to study the existence of solutions of a second order impulsive partial neutral functional integro-differential inclusions with infinite delay:

$$
\begin{aligned}
\frac{d}{d t}\left[x^{\prime}(t)-g\left(t, x_{t}, \int_{0}^{t} a\left(t, s, x_{s}\right) d s\right)\right] & \in A x(t)+F\left(t, x_{t}, \int_{0}^{t} b\left(t, s, x_{s}\right) d s\right), \\
t \in J=[0, T], \quad t \neq t_{k}, \quad k=1,2, \ldots, m, & \quad \quad k(0)=x_{1} \in E, \\
x(t) & =\varphi \in \mathcal{B}_{h}, \quad x^{\prime}(0), \quad k=1,2, \ldots, m, \\
\left.\Delta x\right|_{t=t_{k}} & =I_{k}^{1}\left(x\left(t_{k}^{-}\right)\right), \quad k=1,2, \ldots, m, \\
\left.\Delta x^{\prime}\right|_{t=t_{k}} & =I_{k}^{2}\left(x\left(t_{k}^{-}\right)\right), \quad k=1, \quad
\end{aligned}
$$

where the state variable $x(\cdot)$ takes values in Banach space $E, A$ is the infinitesimal generator of a strongly continuous cosine family $\{C(t): t \in \mathbb{R}\}$ in a real Banach space $E$. The function $F: J \times \mathcal{B}_{h} \times E \rightarrow 2^{E}$ is a bounded, closed, convex-valued map, $g: J \times \mathcal{B}_{h} \times E \rightarrow E, a, b: J \times J \times \mathcal{B}_{h} \rightarrow E, 0=t_{0}<t_{1}<\cdots<t_{m}<t_{m+1}=T$, and $\left.\Delta x\right|_{t=t_{k}}=x\left(t_{k}^{+}\right)-x\left(t_{k}^{-}\right), x\left(t_{k}^{-}\right)$and $x\left(t_{k}^{+}\right)$represent the left and right limits of $x(t)$ at $t=t_{k}$, respectively. The histories $x_{t}:(-\infty, 0] \rightarrow E, x_{t}(s)=x(t+s), s \leq 0$, belong to an abstract phase space $\mathcal{B}_{h}$ which is defined in Section 2 .

\section{Preliminaries}

At first, we present the abstract phase space $\mathcal{B}_{h}$, which has been used in [15]. Assume that $h:(-\infty, 0] \rightarrow(0,+\infty)$ is a continuous function with $\ell=\int_{-\infty}^{0} h(t) d t<+\infty$. For any $e>0$, we define

$$
\mathcal{B}=\{\psi:[-e, 0] \rightarrow E \text { such that } \psi(t) \text { is bounded and measurable }\},
$$

and equip the space $\mathcal{B}$ with the norm

$$
\|\psi\|_{[-e, 0]}=\sup _{s \in[-e, 0]}|\psi(s)|, \quad \forall \psi \in \mathcal{B}
$$


Let us define

$$
\begin{gathered}
\mathcal{B}_{h}=\left\{\psi:(-\infty, 0] \rightarrow E \text { such that for any } c>0,\left.\psi\right|_{[-c, 0]} \in \mathcal{B}\right. \\
\text { and } \left.\int_{-\infty}^{0} h(s)\|\psi\|_{[s, 0]} d s<+\infty\right\} .
\end{gathered}
$$

If $\mathcal{B}_{h}$ is endowed with the norm

$$
\|\psi\|_{\mathcal{B}_{h}}=\int_{-\infty}^{0} h(s)\|\psi\|_{[s, 0]} d s, \forall \psi \in \mathcal{B}_{h},
$$

then it is clear that $\left(\mathcal{B}_{h},\|\cdot\|_{\mathcal{B}_{h}}\right)$ is a Banach space.

Now we consider the space

$$
\begin{aligned}
\mathcal{B}_{h}^{\prime}=\left\{x:(-\infty, T] \rightarrow E \text { such that } x_{k} \in C\left(J_{k}, E\right) \text { and there exist } x\left(t_{k}^{+}\right)\right. \\
\\
\text {and } \left.x\left(t_{k}^{-}\right) \text {with } x\left(t_{k}\right)=x\left(t_{k}^{-}\right), x_{0}=\varphi \in \mathcal{B}_{h}, k=0,1, . ., m\right\},
\end{aligned}
$$

where $x_{k}$ is the restriction of $x$ to $J_{k}=\left(t_{k}, t_{k+1}\right], k=0,1, . ., m$. Set $\|\cdot\|_{T}$ be a seminorm in $\mathcal{B}_{h}^{\prime}$ defined by

$$
\|x\|_{T}=\|\varphi\|_{\mathcal{B}_{h}}+\sup \{|x(s)|: s \in[0, T]\}, x \in \mathcal{B}_{h}^{\prime} .
$$

Next, we introduce definitions, notations, and preliminary facts from multivalued analysis which are used thoughout this paper.

The notation $C(J, E)$ is the Banach space of continuous functions from $J$ into $E$ with the norm $\|x\|_{\infty}=\sup _{t \in J}|x(t)|$ for $x \in C(J, E)$. $B(E)$ denotes the Banach space of bounded linear operator from $E$ into $E$. A measurable function $x: J \rightarrow E$ is Bochner integrable if and only if $|x|$ is Lebesgue integrable. $L^{1}(J, E)$ denotes the Banach space of continuous functions $x: J \rightarrow E$ which are Bochner integrable norm by $\|x\|_{L^{1}}=\int_{0}^{T}|x(t)| d t$ for all $x \in L^{1}(J, E)$.

Let $(E,\|\cdot\|)$ be a Banach space. A multivalued map $\mathcal{F}: E \rightarrow 2^{E}$ is convex (closed) valued, if $\mathcal{F}(x)$ is convex (closed) for all $x \in E$. $\mathcal{F}$ is bounded on bounded set if $\mathcal{F}(B)=\bigcup_{x \in B} \mathcal{F}(x)$ is bounded in $E$, for any bounded set $B$ of $E$ ( i.e., $\sup _{x \in B} \sup \{\|y\| \in \mathcal{F}(x)\}<\infty$ ).

$\mathcal{F}$ is called upper semicontinuous (u.s.c.) on $E$ if for each $x_{*} \in E$, the $\operatorname{set} \mathcal{F}\left(x_{*}\right)$ is nonempty, closed subset of $E$, and if for each open set $B$ of $E$ containing $\mathcal{F}\left(x_{*}\right)$, there exists an open neighbourhood $V$ of $x_{*}$ such that $\mathcal{F}(V) \subset B$.

$\mathcal{F}$ is said to be completely continuous if $\mathcal{F}(B)$ is relatively compact, for every bounded subset $B \subset E$.

If the multivalued map $\mathcal{F}$ is completely continuous with nonempty compact values, then $\mathcal{F}$ is u.s.c. if and only if $\mathcal{F}$ has a closed graph (i.e., $x_{n} \rightarrow x_{*}, y_{n} \rightarrow y_{*}, y_{n} \in \mathcal{F} x_{n}$ imply $y_{*} \in \mathcal{F} x_{*}$ ).

$\mathcal{F}$ has a fixed point if there is $x \in E$ such that $x \in \mathcal{F} x$.

Let $B C C(E)$ denote the set of all nonempty, bounded, closed and convex subsets of $E$. A multivalued map $\mathcal{F}: J \rightarrow B C C(E)$ is said to be measurable if for each $x \in E$ the function $G: J \rightarrow \mathbb{R}$ defined by

$$
G(t)=d(x, \mathcal{F}(t))=\inf \{|x-y|: y \in \mathcal{F}(t)\}
$$

belongs to $L^{1}(J, \mathbb{R})$. For more details on multivalued maps see the books of Deimling [21] and Hu and Papageorgiou [38].

An upper semicontinuous map $H: E \rightarrow E$ is said to be condensing $[6$ if for any subset $D \subset E$ with $\alpha(D) \neq 0$, we have $\alpha(H(D))<\alpha(D)$, where $\alpha$ denotes the Kuratowski measure of noncompactness [6]. It is easy to see that a completely continuous multivalued map is a condensing map.

Throughout this paper, $A: D(A) \subset E \rightarrow E$ is the infinitesimal generator of a strongly continuous cosine function of bounded linear operators $(C(t))_{t \in \mathbb{R}}$ on Banach space $(E,\|\cdot\|)$. We denote by $(S(t))_{t \in \mathbb{R}}$ the sine function associated with $(C(t))_{t \in \mathbb{R}}$ which is defined by $S(t) x=\int_{0}^{t} C(s) x d s$, for $x \in E$ and $t \in \mathbb{R}$. Moreover, $M_{0}$ and $M_{1}$ are positive constants such that $\|C(t)\| \leq M_{0}$ and $\|S(t)\| \leq M_{1}$ for every $t \in J$.

The notation $[D(A)]$ stands for the domain of the operator $A$ endowed with the graph norm $\|x\|_{A}=\|x\|+\|A x\|$, $x \in D(A)$. Moreover, in this work, $\mathbb{E}$ is the space formed by the vectors $x \in E$ for which $C(\cdot) x$ is of class $C^{1}$ on $\mathbb{R}$. It was proved by Kisinsky [40] that $\mathbb{E}$ endowed with the norm

$$
\|x\|_{\mathbb{E}}=\|x\|+\sup _{0 \leq t \leq 1}\|A S(t) x\|, \quad x \in \mathbb{E},
$$

is a Banach space. The operator valued function $G(t)=\left[\begin{array}{ll}C(t) & S(t) \\ A S(t) & C(t)\end{array}\right]$ is a strongly continuous group of bounded linear operators on the space $E \times X$ generated by the operator $\mathcal{A}=\left[\begin{array}{cc}0 & I \\ A & 0\end{array}\right]$ defined on $D(A) \times \mathbb{E}$. It follows from 
this that $A S(t): \mathbb{E} \rightarrow E$ is a bounded linear operator and that $A S(t) x \rightarrow 0, t \rightarrow 0$, for each $x \in \mathbb{E}$. Furthermore, if $x:[0, \infty) \rightarrow X$ is a locally integrable function, then $z(t)=\int_{0}^{t} S(t-s) x(s) d s$ defines an $\mathbb{E}$-valued continuous function. This is a consequence of the fact that

$$
\int_{0}^{t} G(t-s)\left[\begin{array}{c}
0 \\
x(s)
\end{array}\right] d s=\left[\int_{0}^{t} S(t-s) x(s) d s, \quad \int_{0}^{t} C(t-s) x(s) d s\right]^{T}
$$

defines an $\mathbb{E} \times E$-valued continuous function.

The existence of solutions for the second order abstract Cauchy problem

$$
\left\{\begin{aligned}
x^{\prime \prime}(t) & =A x(t)+h(t), \quad 0 \leq t \leq T \\
x(0) & =z, \quad x^{\prime}(0)=w
\end{aligned}\right.
$$

where $h: I \rightarrow E$ is an integrable function has been discussed in [52. Similarly, the existence of solutions of the semilinear second order abstract Cauchy problem it has been treated in [53. We only mention here that the function $x(\cdot)$ given by

$$
x(t)=C(t) z+S(t) w+\int_{0}^{t} S(t-s) h(s) d s, \quad 0 \leq t \leq T,
$$

is called mild solution of $(2.1)$ and that when $z \in \mathbb{E}, x(\cdot)$ is continuously differentiable and

$$
x^{\prime}(t)=A S(t) z+C(t) w+\int_{0}^{t} C(t-s) h(s) d s, \quad 0 \leq t \leq T .
$$

For additional details about cosine function theory, we refer to the reader to [52, 53].

For our approach, we need the following fixed point theorem.

Theorem 2.1 (Martelli [48]). Let $E$ be a Banach space and $\Phi: E \rightarrow B C C(E)$ a condensing map. If the set

$$
\Lambda=\{x \in E: \lambda x \in \Phi x, \text { for some } \lambda>1\}
$$

is bounded then $\Phi$ has a fixed point.

\section{Existence Results}

In this section, we shall present and prove existence results for the problem (1.1)-(1.4). First, we give the mild solution for the problem (1.1)-(1.4).

Definition 3.1. A function $x:(-\infty, T] \rightarrow E$ is called a mild solution of problem (1.1)-(1.4) if the following holds: $x_{0}=\varphi \in \mathcal{B}_{h}$ on $(-\infty, 0], x^{\prime}(0)=x_{1} ;\left.\Delta x\right|_{t=t_{k}}=I_{k}^{1}\left(x\left(t_{k}^{-}\right)\right), k=1,2, \ldots, m,\left.\Delta x^{\prime}\right|_{t=t_{k}}=I_{k}^{2}\left(x\left(t_{k}^{-}\right)\right), k=1,2, \ldots, m$, the restriction of $x(\cdot)$ to the interval $[0, T)-\left\{t_{1}, t_{2}, \ldots, t_{m}\right\}$ is continuous, and for each $s \in[0, t)$, the impulsive integral equation

$$
\begin{aligned}
x(t)= & C(t) \varphi(0)+S(t)\left[x_{1}-g(0, \varphi, 0)\right]+\int_{0}^{t} C(t-s) g\left(s, x_{s}, \int_{0}^{s} a\left(s, \tau, x_{\tau}\right) d \tau\right) d s \\
& +\int_{0}^{t} S(t-s) f(s) d s+\sum_{0<t_{k}<t} C\left(t-t_{k}\right) I_{k}^{1}\left(x\left(t_{k}^{-}\right)\right)+\sum_{0<t_{k}<t} S\left(t-t_{k}\right) I_{k}^{2}\left(x\left(t_{k}^{-}\right)\right), \quad t \in J
\end{aligned}
$$

is satisfied, where

$$
f \in S_{F, x}=\left\{f \in L^{1}(J, E): f(t) \in F\left(t, x_{t}, \int_{0}^{t} b\left(t, s, x_{s}\right) d s\right) \text {, for a.e. } t \in J\right\} .
$$

For the study of the problem (1.1)-(1.4), we need the following hypotheses:

(H1) (i) There exist a constant $L>0$ such that

$$
\left\|\int_{0}^{t}[a(t, s, x)-a(t, s, y)] d s\right\| \leq L\|x-y\|_{\mathcal{B}_{h}} \quad \text { for } \quad t, s \in J, \quad x, y \in \mathcal{B}_{h} .
$$


(ii) There exist constants $L_{1}, \widetilde{L}_{1}$ such that

$$
\left\|\int_{0}^{t} a(t, s, x) d s\right\| \leq L_{1}\|x\|_{\mathcal{B}_{h}}+\widetilde{L}_{1}, \quad t, s \in J, \quad x \in \mathcal{B}_{h} .
$$

(H2) (i) The function $g: J \times \mathcal{B}_{h} \times E \rightarrow E$ is continuous and there exists a constant $L_{2}>0$ such that the function $g$ satisfies the Lipschitz condition:

$$
\begin{gathered}
\left\|g\left(t_{1}, x_{1}, x_{2}\right)-g\left(t_{2}, y_{1}, y_{2}\right)\right\| \leq L_{2}\left[\left\|t_{1}-t_{2}\right\|+\left\|x_{1}-y_{1}\right\|_{\mathcal{B}_{h}}+\left\|x_{2}-y_{2}\right\|\right] \\
t_{1}, t_{2} \in J, x_{1}, y_{1} \in \mathcal{B}_{h}, x_{2}, y_{2} \in E
\end{gathered}
$$

(ii) There exist constants $L_{3}, \widetilde{L}_{3}$ such that $\ell L_{3}<1$ and

$$
\|g(t, x, y)\| \leq L_{3}\left[\|x\|_{\mathcal{B}_{h}}+\|y\|\right]+\widetilde{L}_{3}, t \in J, x \in \mathcal{B}_{h}, y \in E
$$

where $\ell=\int_{-\infty}^{0} h(s) d s<+\infty$.

(H3) (i) $F: J \times \mathcal{B}_{h} \times E \rightarrow B C C(E) ;(t, x, y) \rightarrow F(t, x, y)$ is measurable with respect to $t$ for each $x \in \mathcal{B}_{h}, y \in E$, u.s.c. with respect to $x, y$ for each $t \in J$, and for each fixed $x \in \mathcal{B}_{h}, y \in E$, the set

$$
S_{F, x}=\left\{f \in L^{1}(J, E): f(t) \in F\left(t, x_{t}, \int_{0}^{t} b\left(t, s, x_{s}\right) d s\right), \text { for a.e. } t \in J\right\} .
$$

is nonempty.

(ii) There exists an integrable function $m: J \rightarrow[0, \infty)$ such that

$$
\begin{aligned}
\left\|F\left(t, x_{t}, \int_{0}^{t} b\left(t, s, x_{s}\right) d s\right)\right\| & =\sup \left\{|f|: f \in F\left(t, x_{t}, \int_{0}^{t} b\left(t, s, x_{s}\right) d s\right)\right\} \\
& \leq m(t) \Omega\left(\|x\|_{\mathcal{B}_{h}}+\|y\|\right), t \in J, x \in \mathcal{B}_{h}, y \in E
\end{aligned}
$$

where $\Omega:[0, \infty) \rightarrow(0, \infty)$ is a continuous nondecreasing function.

(H4) For each $(t, s) \in J \times J$, the function $b(t, s, \cdot): \mathcal{B}_{h} \rightarrow E$ is continuous and for each $x \in \mathcal{B}_{h}$, the function $b(\cdot, \cdot, x): J \times J \rightarrow E$ is strongly measurable. There exist an integrable function $p: J \rightarrow[0, \infty)$ and a constant $\gamma>0$, such that

$$
\|b(t, s, x)\| \leq \gamma p(s) \Theta\left(\|x\|_{\mathcal{B}_{h}}\right)
$$

where $\Theta:[0, \infty) \rightarrow(0, \infty)$ is a continuous nondecreasing function. Assume that the finite bound of $\int_{0}^{t} \gamma p(s) d s$ is $L_{0}$.

(H5) $I_{k}^{1}, I_{k}^{2} \in C(E, E)$ and there exist constant $d_{k}, \tilde{d}_{k}$ such that $\left\|I_{k}^{1}(x)\right\| \leq d_{k},\left\|I_{k}^{2}(x)\right\| \leq \tilde{d}_{k}, k=1,2, \ldots, m$ for each $x \in E$.

(H6) The following inequality holds:

$$
\int_{0}^{T} \widetilde{m}(s) d s<\int_{h_{1}}^{\infty} \frac{d s}{s+\Omega(s)+\Theta(s)},
$$

where $h_{1}=\|\varphi\|_{\mathcal{B}_{h}}+\ell K_{1}, h_{2}=\ell M_{0} L_{3}\left(1+L_{1}\right), h_{3}=\ell M_{1}, \tilde{m}(t)=\max \left\{h_{2}, h_{3} m(t), \gamma p(t)\right\}$, $t \in J$, and $K_{1}=M_{0}\left[|\varphi(0)|+T\left(L_{3} \widetilde{L}_{1}+\widetilde{L}_{3}\right)+\sum_{k=1}^{m} d_{k}\right]+M_{1}\left[\left|x_{1}\right|+L_{3}\|\varphi\|_{\mathcal{B}_{h}}+\widetilde{L}_{3}+\sum_{k=1}^{m} \tilde{d}_{k}\right]$.

Remark 3.2. (i) If $\operatorname{dim} E<\infty$, then for each $x \in \mathcal{B}_{h}, S_{F, x} \neq \emptyset$ ( See [44]).

(ii) $S_{F, x}$ is nonempty if and only if the function $Y: J \rightarrow \mathbb{R}$ defined by $Y(t)=\inf \{|f|: f \in F(t, x, y)\}$ belongs to $L^{1}(J, \mathbb{R})$.

Lemma 3.3. (Lasota and Opial [44]). Let $J$ be a compact real interval and $E$ be a Banach space. Let $F$ be a multi-valued map satisfying (H2)(i) and let $\Gamma$ be a linear continuous mapping from $L^{1}(J, E)$ to $C(J, E)$. Then the operator

$$
\Gamma \circ S_{F}: C(J, \mathbb{X}) \rightarrow B C C(C(J, E)), \quad x \mapsto\left(\Gamma \circ S_{F}\right)(x):=\Gamma\left(S_{F, x}\right)
$$

is a closed graph operator in $C(J, E) \times C(J, E)$. 
Lemma 3.4. [18]. Assume $x \in \mathcal{B}_{h}^{\prime}$, then for $t \in J, x_{t} \in \mathcal{B}_{h}$. Moreover,

$$
\ell|x(t)| \leq\left\|x_{t}\right\|_{\mathcal{B}_{h}} \leq\left\|x_{0}\right\|_{\mathcal{B}_{h}}+\ell \sup _{s \in[0, t]}|x(s)|,
$$

where $\ell=\int_{-\infty}^{0} h(t) d t<+\infty$.

Consider the multivalued map $\Phi: \mathcal{B}_{h}^{\prime} \rightarrow 2^{\mathcal{B}_{h}^{\prime}}$ defined by $\Phi x$ the set of $\rho \in \mathcal{B}_{h}^{\prime}$ such that

$$
\rho(t)=\left\{\begin{array}{l}
\varphi(t), \text { if } t \in(-\infty, 0] \\
C(t) \varphi(0)+S(t)\left[x_{1}-g(0, \varphi, 0)\right]+\int_{0}^{t} C(t-s) g\left(s, x_{s}, \int_{0}^{s} a\left(s, \tau, x_{\tau}\right) d \tau\right) d s \\
+\int_{0}^{t} S(t-s) f(s) d s+\sum_{0<t_{k}<t} C\left(t-t_{k}\right) I_{k}^{1}\left(x\left(t_{k}^{-}\right)\right)+\sum_{0<t_{k}<t} S\left(t-t_{k}\right) I_{k}^{2}\left(x\left(t_{k}^{-}\right)\right), \quad t \in J
\end{array}\right.
$$

where $f \in S_{F, x}$.

We shall show that the operators $\Phi$ has fixed points, which are then a solution of equations (1.1)-(1.4). Clearly, $x_{1} \in(\Phi x)(T)$.

For $\varphi \in \mathcal{B}_{h}$, we define $\widetilde{\varphi}$ by

$$
\tilde{\varphi}(t)= \begin{cases}\varphi(t), & t \in(-\infty, 0] \\ C(t) \varphi(0), & t \in J\end{cases}
$$

then $\tilde{\varphi} \in \mathcal{B}_{h}^{\prime}$. Let $x(t)=y(t)+\tilde{\varphi}(t),-\infty<t \leq T$. It is easy to see that $x$ satisfies (3.1) if and only if $y$ satisfies $y_{0}=0, x^{\prime}(0)=x_{1}=y^{\prime}(0)=y_{1}$ and

$$
\begin{aligned}
y(t)= & S(t)\left[y_{1}-g(0, \varphi, 0)\right]+\int_{0}^{t} C(t-s) g\left(s, y_{s}+\tilde{\varphi}_{s}, \int_{0}^{s} a\left(s, \tau, y_{\tau}+\tilde{\varphi}_{\tau}\right) d \tau\right) d s \\
& +\int_{0}^{t} S(t-s) f(s) d s+\sum_{0<t_{k}<t} C\left(t-t_{k}\right) I_{k}^{1}\left(y\left(t_{k}^{-}\right)+\tilde{\varphi}\left(t_{k}^{-}\right)\right) \\
& +\sum_{0<t_{k}<t} S\left(t-t_{k}\right) I_{k}^{2}\left(y\left(t_{k}^{-}\right)+\tilde{\varphi}\left(t_{k}^{-}\right)\right), \quad t \in J .
\end{aligned}
$$

Let $\mathcal{B}_{h}^{\prime \prime}=\left\{y \in \mathcal{B}_{h}^{\prime}: y_{0}=0 \in \mathcal{B}_{h}\right\}$. For any $y \in \mathcal{B}_{h}^{\prime \prime}$,

$$
\begin{aligned}
\|y\|_{T} & =\left\|y_{0}\right\|_{\mathcal{B}_{h}}+\sup \{|y(s)|: 0 \leq s \leq T\} \\
& =\sup \{|y(s)|: 0 \leq s \leq T\}
\end{aligned}
$$

thus $\left(\mathcal{B}_{h}^{\prime \prime},\|\cdot\|_{T}\right)$ is a Banach space. Set $B_{r}=\left\{y \in \mathcal{B}_{h}^{\prime \prime}:\|y\|_{T} \leq r\right\}$ for some $r \geq 0$, then $B_{r} \subseteq \mathcal{B}_{h}^{\prime \prime}$ is uniformly bounded, and for $y \in B_{r}$, from Lemma 3.4, we have

$$
\begin{aligned}
\left\|y_{t}+\tilde{\varphi}_{t}\right\|_{\mathcal{B}_{h}} & \leq\left\|y_{t}\right\|_{\mathcal{B}_{h}}+\left\|\tilde{\varphi}_{t}\right\|_{\mathcal{B}_{h}} \\
& \leq \ell \sup _{s \in[0, t]}|y(s)|+\left\|y_{0}\right\|_{\mathcal{B}_{h}}+\ell \sup _{s \in[0, t]}|\tilde{\varphi}(s)|+\left\|\tilde{\varphi}_{0}\right\|_{\mathcal{B}_{h}} \\
& \leq \ell\left(r+M_{0}|\varphi(0)|\right)+\|\varphi\|_{\mathcal{B}_{h}}=r^{\prime} .
\end{aligned}
$$

Define the multivalued map $\Phi_{1}: \mathcal{B}_{h}^{\prime \prime} \rightarrow 2^{\mathcal{B}_{h}^{\prime \prime}}$ defined by $\Phi_{1} y$ the set of $\bar{\rho} \in \mathcal{B}_{h}^{\prime \prime}$ such that

$$
\bar{\rho}(t)=\left\{\begin{array}{l}
0, \text { if } t \in(-\infty, 0] \\
S(t)\left[y_{1}-g(0, \varphi, 0)\right]+\int_{0}^{t} C(t-s) g\left(s, y_{s}+\tilde{\varphi}_{s}, \int_{0}^{s} a\left(s, \tau, y_{\tau}+\tilde{\varphi}_{\tau}\right) d \tau\right) d s \\
+\int_{0}^{t} S(t-s) f(s) d s+\sum_{0<t_{k}<t} C\left(t-t_{k}\right) I_{k}^{1}\left(y\left(t_{k}^{-}\right)+\tilde{\varphi}\left(t_{k}^{-}\right)\right) \\
+\sum_{0<t_{k}<t} S\left(t-t_{k}\right) I_{k}^{2}\left(y\left(t_{k}^{-}\right)+\tilde{\varphi}\left(t_{k}^{-}\right)\right), \quad t \in J
\end{array}\right.
$$

where $f \in S_{F, x}$. 
Lemma 3.5. If the hypotheses (H1)-(H5) are satisfied, then $\Phi_{1}: \mathcal{B}_{h}^{\prime \prime} \rightarrow 2^{\mathcal{B}_{h}^{\prime \prime}}$ is a completely continuous multivalued, u.s.c. with a convex closed value.

Proof. We divide the proof into several steps.

Step 1: $\Phi_{1} y$ is convex for each $y \in \mathcal{B}_{h}^{\prime \prime}$.

In fact, if $\bar{\rho}_{1}, \bar{\rho}_{2}$ belong to $\Phi_{1} y$, then there exist $f_{1}, f_{2} \in S_{F, y}$ such that for each $t \in J$, we have

$$
\begin{aligned}
\bar{\rho}_{i}(t)= & S(t)\left[y_{1}-g(0, \varphi, 0)\right]+\int_{0}^{t} C(t-s) g\left(s, y_{s}+\tilde{\varphi}_{s}, \int_{0}^{s} a\left(s, \tau, y_{\tau}+\tilde{\varphi}_{\tau}\right) d \tau\right) d s \\
& +\int_{0}^{t} S(t-s) f_{i}(s) d s+\sum_{0<t_{k}<t} C\left(t-t_{k}\right) I_{k}^{1}\left(y\left(t_{k}^{-}\right)+\tilde{\varphi}\left(t_{k}^{-}\right)\right) \\
& +\sum_{0<t_{k}<t} S\left(t-t_{k}\right) I_{k}^{2}\left(y\left(t_{k}^{-}\right)+\tilde{\varphi}\left(t_{k}^{-}\right)\right), i=1,2 .
\end{aligned}
$$

Let $\lambda \in[0,1]$, we have

$$
\begin{aligned}
& \left(\lambda \bar{\rho}_{1}+(1-\lambda) \bar{\rho}_{2}\right)(t) \\
& =S(t)\left[y_{1}-g(0, \varphi, 0)\right]+\int_{0}^{t} C(t-s) g\left(s, y_{s}+\tilde{\varphi}_{s}, \int_{0}^{s} a\left(s, \tau, y_{\tau}+\tilde{\varphi}_{\tau}\right) d \tau\right) d s \\
& \quad+\int_{0}^{t} S(t-s)\left[\lambda f_{1}(s)+(1-\lambda) f_{2}(s)\right] d s+\sum_{0<t_{k}<t} C\left(t-t_{k}\right) I_{k}^{1}\left(y\left(t_{k}^{-}\right)+\tilde{\varphi}\left(t_{k}^{-}\right)\right) \\
& \quad+\sum_{0<t_{k}<t} S\left(t-t_{k}\right) I_{k}^{2}\left(y\left(t_{k}^{-}\right)+\tilde{\varphi}\left(t_{k}^{-}\right)\right) .
\end{aligned}
$$

Since $S_{F, y}$ is convex ( because $F$ has convex values), we have $\lambda \bar{\rho}_{1}+(1-\lambda) \bar{\rho}_{2} \in \Phi_{1} y$.

Step 2: $\Phi_{1}$ maps bounded sets into bounded sets in $\mathcal{B}_{h}^{\prime \prime}$.

Indeed, it is enough to show that there exists a positive constant $\mathcal{K}$ such that for each $\bar{\rho} \in \Phi_{1} y, y \in B_{r}=\{y \in$ $\left.\mathcal{B}_{h}^{\prime \prime}:\|y\|_{T} \leq r\right\}$, one has $\|\bar{\rho}\|_{T} \leq \mathcal{K}$.

If $\bar{\rho} \in \Phi_{1} y$, then there exists $f \in S_{F, y}$ such that for each $t \in J$, we have

$$
\begin{aligned}
\bar{\rho}(t)= & S(t)\left[y_{1}-g(0, \varphi, 0)\right]+\int_{0}^{t} C(t-s) g\left(s, y_{s}+\tilde{\varphi}_{s}, \int_{0}^{s} a\left(s, \tau, y_{\tau}+\tilde{\varphi}_{\tau}\right) d \tau\right) d s \\
& +\int_{0}^{t} S(t-s) f(s) d s+\sum_{0<t_{k}<t} C\left(t-t_{k}\right) I_{k}^{1}\left(y\left(t_{k}^{-}\right)+\tilde{\varphi}\left(t_{k}^{-}\right)\right) \\
& +\sum_{0<t_{k}<t} S\left(t-t_{k}\right) I_{k}^{2}\left(y\left(t_{k}^{-}\right)+\tilde{\varphi}\left(t_{k}^{-}\right)\right) .
\end{aligned}
$$

By (H1)-(H5), (3.2) and (3.3), we have for $t \in J$,

$$
\begin{aligned}
|\bar{\rho}(t)| \leq & \left|S(t)\left[y_{1}-g(0, \varphi, 0)\right]\right|+\int_{0}^{t}\left|C(t-s) g\left(s, y_{s}+\tilde{\varphi}_{s}, \int_{0}^{s} a\left(s, \tau, y_{\tau}+\tilde{\varphi}_{\tau}\right) d \tau\right)\right| d s \\
& +\int_{0}^{t}|S(t-s) f(s)| d s+\sum_{0<t_{k}<t}\left|C\left(t-t_{k}\right) I_{k}^{1}\left(y\left(t_{k}^{-}\right)+\tilde{\varphi}\left(t_{k}^{-}\right)\right)\right| \\
& +\sum_{0<t_{k}<t}\left|S\left(t-t_{k}\right) I_{k}^{2}\left(y\left(t_{k}^{-}\right)+\tilde{\varphi}\left(t_{k}^{-}\right)\right)\right| \\
\leq & M_{1}\left|y_{1}\right|+M_{1}|g(0, \varphi, 0)|+M_{0} \int_{0}^{t}\left[L_{3}\left[\left\|y_{s}+\tilde{\varphi}_{s}\right\|_{\mathcal{B}_{h}}+\left\|\int_{0}^{s} a\left(s, \tau, y_{\tau}+\tilde{\varphi}_{\tau}\right) d \tau\right\|\right]+\widetilde{L}_{3}\right] d s \\
& +M_{1} \int_{0}^{t} m(s) \Omega\left(\left\|y_{s}+\tilde{\varphi}_{s}\right\| \mathcal{B}_{h}+\left\|\int_{0}^{s} b\left(s, \tau, y_{\tau}+\tilde{\varphi}_{\tau}\right) d \tau\right\|\right) d s+M_{0} \sum_{k=1}^{m} d_{k}+M_{1} \sum_{k=1}^{m} \tilde{d}_{k} \\
\leq & M_{1}\left|y_{1}\right|+M_{1}|g(0, \varphi, 0)|+M_{0} T\left[L_{3} r^{\prime}+\widetilde{L}_{3}+L_{3}\left(L_{1} r^{\prime}+\widetilde{L}_{1}\right)\right] \\
& +M_{1} \Omega\left[r^{\prime}+L_{0} \Theta\left(r^{\prime}\right)\right] \int_{0}^{T} m(s) d s+M_{0} \sum_{k=1}^{m} d_{k}+M_{1} \sum_{k=1}^{m} \tilde{d}_{k} \\
= & \mathcal{K} .
\end{aligned}
$$


Thus, for each $\bar{\rho} \in \Phi_{1}\left(B_{r}\right)$, we obtain $\|\bar{\rho}\|_{T} \leq \mathcal{K}$.

Step 3: $\Phi_{1}$ maps bounded sets into equicontinuous sets of $\mathcal{B}_{h}^{\prime \prime}$.

Let $0<\tau_{1}<\tau_{2} \leq T-\left\{t_{1}, t_{2}, \ldots, t_{m}\right\}$, for each $\bar{\rho} \in \Phi_{1} y, y \in B_{r}=\left\{y \in \mathcal{B}_{h}^{\prime \prime}:\|y\|_{T} \leq r\right\}$ and $\bar{\rho} \in \Phi_{1} y$, there exists $f \in S_{F, y}$ satisfying (3.3). Thus, we see that

$$
\begin{aligned}
& \left|\bar{\rho}\left(\tau_{2}\right)-\bar{\rho}\left(\tau_{1}\right)\right| \\
& \leq\left|\left[S\left(\tau_{2}\right)-S\left(\tau_{1}\right)\right]\left[y_{1}-g(0, \varphi, 0)\right]\right| \\
& +\int_{0}^{\tau_{1}}\left|\left[C\left(\tau_{2}-s\right)-C\left(\tau_{1}-s\right)\right] g\left(s, y_{s}+\tilde{\varphi}_{s}, \int_{0}^{s} a\left(s, \tau, y_{\tau}+\tilde{\varphi}_{\tau}\right) d \tau\right)\right| d s \\
& +\int_{\tau_{1}}^{\tau_{2}}\left|C\left(\tau_{2}-s\right) g\left(s, y_{s}+\tilde{\varphi}_{s}, \int_{0}^{s} a\left(s, \tau, y_{\tau}+\tilde{\varphi}_{\tau}\right) d \tau\right)\right| d s \\
& +\int_{0}^{\tau_{1}}\left|\left[S\left(\tau_{2}-s\right)-S\left(\tau_{1}-s\right)\right] f(s)\right| d s \\
& +\int_{\tau_{1}}^{\tau_{2}}\left|S\left(\tau_{2}-s\right) f(s)\right| d s+\sum_{0<t_{k}<\tau_{1}}\left|\left[C\left(\tau_{2}-t_{k}\right)-C\left(\tau_{1}-t_{k}\right)\right] I_{k}^{1}\left(y\left(t_{k}^{-}\right)+\tilde{\varphi}\left(t_{k}^{-}\right)\right)\right| \\
& +\sum_{\tau_{1}<t_{k}<\tau_{2}}\left|C\left(\tau_{2}-t_{k}\right) I_{k}^{1}\left(y\left(t_{k}^{-}\right)+\tilde{\varphi}\left(t_{k}^{-}\right)\right)\right| \\
& +\sum_{0<t_{k}<\tau_{1}}\left|\left[S\left(\tau_{2}-t_{k}\right)-S\left(\tau_{1}-t_{k}\right)\right] I_{k}^{2}\left(y\left(t_{k}^{-}\right)+\tilde{\varphi}\left(t_{k}^{-}\right)\right)\right| \\
& +\sum_{\tau_{1}<t_{k}<\tau_{2}}\left|S\left(\tau_{2}-t_{k}\right) I_{k}^{2}\left(y\left(t_{k}^{-}\right)+\tilde{\varphi}\left(t_{k}^{-}\right)\right)\right| \\
& \leq\left|S\left(\tau_{2}\right)-S\left(\tau_{1}\right)\right|\left|y_{1}-g(0, \varphi, 0)\right| \\
& +\int_{0}^{\tau_{1}}\left[\left|C\left(\tau_{2}-s\right)-C\left(\tau_{1}-s\right)\right| L_{3}\left[\left\|y_{s}+\tilde{\varphi}_{s}\right\|_{\mathcal{B}_{h}}+L_{1}\left(\left\|y_{s}+\tilde{\varphi}_{s}\right\|_{\mathcal{B}_{h}}+\widetilde{L}_{1}\right)\right]+\widetilde{L}_{3}\right] d s \\
& \left.+\int_{\tau_{1}}^{\tau_{2}}\left|C\left(\tau_{2}-s\right)\right| L_{3}\left[\left\|y_{s}+\tilde{\varphi}_{s}\right\|_{\mathcal{B}_{h}}+L_{1}\left(\left\|y_{s}+\tilde{\varphi}_{s}\right\|_{\mathcal{B}_{h}}+\widetilde{L}_{1}\right)\right]+\widetilde{L}_{3}\right] d s \\
& +\int_{0}^{\tau_{1}}\left|S\left(\tau_{2}-s\right)-S\left(\tau_{1}-s\right) \| f(s)\right| d s \\
& +\int_{\tau_{1}}^{\tau_{2}}\left|S\left(\tau_{2}-s\right)\right||f(s)| d s+\sum_{0<t_{k}<\tau_{1}}\left|C\left(\tau_{2}-t_{k}\right)-C\left(\tau_{1}-t_{k}\right)\right| d_{k}+\sum_{\tau_{1}<t_{k}<\tau_{2}}\left|C\left(\tau_{2}-t_{k}\right)\right| d_{k} \\
& +\sum_{0<t_{k}<\tau_{1}}\left|S\left(\tau_{2}-t_{k}\right)-S\left(\tau_{1}-t_{k}\right)\right| \tilde{d}_{k}+\sum_{\tau_{1}<t_{k}<\tau_{2}}\left|S\left(\tau_{2}-t_{k}\right)\right| \tilde{d}_{k} .
\end{aligned}
$$

The right hand side of above inequality is independent of $y \in B_{r}$ and tends to zero as $\tau_{2}-\tau_{1} \rightarrow 0$. Thus the set $\left\{\Phi_{1} y: y \in B_{r}\right\}$ is equicontinuous (Note that this proves the equicontinuity for the case where $t \neq t_{k}, k=1,2, \ldots, m+1$. Easily we prove the equicontinuity for the case where $t=t_{i}$. And also the other cases $\tau_{1}<\tau_{2} \leq 0$ or $\tau_{1} \leq 0 \leq \tau_{2} \leq T$ are very simple).

As a consequence of steps 2 and 3 together with the Arzela-Ascoli theorem we can conclude that $\Phi_{1}: \mathcal{B}_{h}^{\prime \prime} \rightarrow 2^{\mathcal{B}_{h}^{\prime \prime}}$ is a compact multivalued map, and therefore, a condensing map.

Step 4: $\Phi_{1}$ has a closed graph.

Let $y_{n} \rightarrow y_{*}, \bar{\rho}_{n} \in \Phi_{1} y_{n}$ and $\bar{\rho}_{n} \rightarrow \bar{\rho}_{*}$. We shall prove that $\bar{\rho}_{*} \in \Phi_{1} y_{*}$. Indeed, $\bar{\rho}_{n} \in \Phi_{1} y_{n}$ means that there exists $f_{n} \in S_{F, y_{n}}$ such that

$$
\begin{aligned}
\bar{\rho}_{n}(t)= & S(t)\left[y_{1}-g(0, \varphi, 0)\right]+\int_{0}^{t} C(t-s) g\left(s, y_{n_{s}}+\tilde{\varphi}_{s}, \int_{0}^{s} a\left(s, \tau, y_{n_{\tau}}+\tilde{\varphi}_{\tau}\right) d \tau\right) d s \\
& +\int_{0}^{t} S(t-s) f_{n}(s) d s+\sum_{0<t_{k}<t} C\left(t-t_{k}\right) I_{k}^{1}\left(y_{n}\left(t_{k}^{-}\right)+\tilde{\varphi}\left(t_{k}^{-}\right)\right) \\
& +\sum_{0<t_{k}<t} S\left(t-t_{k}\right) I_{k}^{2}\left(y_{n}\left(t_{k}^{-}\right)+\tilde{\varphi}\left(t_{k}^{-}\right)\right), t \in J .
\end{aligned}
$$


We must prove that there exists $f_{*} \in S_{F, y_{*}}$ such that

$$
\begin{aligned}
\bar{\rho}_{*}(t)= & S(t)\left[y_{1}-g(0, \varphi, 0)\right]+\int_{0}^{t} C(t-s) g\left(s, y_{*_{s}}+\tilde{\varphi}_{s} \int_{0}^{s} a\left(s, \tau, y_{*_{\tau}}+\tilde{\varphi}_{\tau}\right) d \tau\right) d s \\
& +\int_{0}^{t} S(t-s) f_{*}(s) d s+\sum_{0<t_{k}<t} C\left(t-t_{k}\right) I_{k}^{1}\left(y_{*}\left(t_{k}^{-}\right)+\tilde{\varphi}\left(t_{k}^{-}\right)\right) \\
& +\sum_{0<t_{k}<t} S\left(t-t_{k}\right) I_{k}^{2}\left(y_{*}\left(t_{k}^{-}\right)+\tilde{\varphi}\left(t_{k}^{-}\right)\right), t \in J .
\end{aligned}
$$

Then

$$
\begin{aligned}
& \|\left\{\bar{\rho}_{n}(t)-S(t)\left[y_{1}-g(0, \varphi, 0)\right]-\int_{0}^{t} C(t-s) g\left(s, y_{n_{s}}+\tilde{\varphi}_{s}, \int_{0}^{s} a\left(s, \tau, y_{n_{\tau}}+\tilde{\varphi}_{\tau}\right) d \tau\right) d s\right. \\
& \left.\quad-\sum_{0<t_{k}<t} C\left(t-t_{k}\right) I_{k}^{1}\left(y_{n}\left(t_{k}^{-}\right)+\tilde{\varphi}\left(t_{k}^{-}\right)\right)-\sum_{0<t_{k}<t} S\left(t-t_{k}\right) I_{k}^{2}\left(y_{n}\left(t_{k}^{-}\right)+\tilde{\varphi}\left(t_{k}^{-}\right)\right)\right\} \\
& -\left\{\bar{\rho}_{*}(t)-S(t)\left[y_{1}-g(0, \varphi, 0)\right]-\int_{0}^{t} C(t-s) g\left(s, y_{*_{s}}+\tilde{\varphi}_{s}, \int_{0}^{s} a\left(s, \tau, y_{*_{\tau}}+\tilde{\varphi}_{\tau}\right) d \tau\right) d s\right. \\
& \left.\quad-\sum_{0<t_{k}<t} C\left(t-t_{k}\right) I_{k}^{1}\left(y_{*}\left(t_{k}^{-}\right)+\tilde{\varphi}\left(t_{k}^{-}\right)\right)-\sum_{0<t_{k}<t} S\left(t-t_{k}\right) I_{k}^{2}\left(y_{*}\left(t_{k}^{-}\right)+\tilde{\varphi}\left(t_{k}^{-}\right)\right)\right\} \|_{T} \\
& \rightarrow 0 \text { as } n \rightarrow \infty .
\end{aligned}
$$

Consider the linear operator $\Gamma: L^{1}(J, E) \rightarrow C(J, E)$ defined by

$$
f \rightarrow \Gamma(f)(t)=\int_{0}^{t} S(t-s) f(s) d s .
$$

Clearly, $\Gamma$ is linear and continuous. Indeed, one has

$$
\|\Gamma f\|_{\infty} \leq M_{1}\|f\|_{L^{1}} .
$$

From Lemma 3.3, it follws that $\Gamma \circ S_{F}$ is a closed graph operator. Moreover, we have

$$
\begin{aligned}
& \bar{\rho}_{n}(t)-S(t)\left[y_{1}-g(0, \varphi)\right]-\int_{0}^{t} C(t-s) g\left(s, y_{n_{s}}+\tilde{\varphi}_{s}, \int_{0}^{s} a\left(s, \tau, y_{n_{\tau}}+\tilde{\varphi}_{\tau}\right) d \tau\right) d s \\
& \quad-\sum_{0<t_{k}<t} C\left(t-t_{k}\right) I_{k}^{1}\left(y_{n}\left(t_{k}^{-}\right)+\tilde{\varphi}\left(t_{k}^{-}\right)\right)-\sum_{0<t_{k}<t} S\left(t-t_{k}\right) I_{k}^{2}\left(y_{n}\left(t_{k}^{-}\right)+\tilde{\varphi}\left(t_{k}^{-}\right)\right) \in \Gamma\left(S_{F, y_{n}}\right) .
\end{aligned}
$$

Since $y_{n} \rightarrow y_{*}$, it follows from Lemma 3.3 that

$$
\begin{aligned}
\bar{\rho}_{*}(t) & -S(t)\left[y_{1}-g(0, \varphi)\right]-\int_{0}^{t} C(t-s) g\left(s, y_{*_{s}}+\tilde{\varphi}_{s}, \int_{0}^{s} a\left(s, \tau, y_{*_{\tau}}+\tilde{\varphi}_{\tau}\right) d \tau\right) d s \\
& -\sum_{0<t_{k}<t} C\left(t-t_{k}\right) I_{k}^{1}\left(y_{*}\left(t_{k}^{-}\right)+\tilde{\varphi}\left(t_{k}^{-}\right)\right)-\sum_{0<t_{k}<t} S\left(t-t_{k}\right) I_{k}^{2}\left(y_{*}\left(t_{k}^{-}\right)+\tilde{\varphi}\left(t_{k}^{-}\right)\right) \\
& =\int_{0}^{t} S(t-s) f_{*}(s) d s
\end{aligned}
$$

for some $f_{*} \in S_{F, y_{*}}$.

Hence $\Phi_{1}$ is a completely continuous multivalued map, u.s.c. with convex closed values.

Now in order to apply Theorem 2.1, we introduce a parameter $\lambda>1$ and consider the following equation:

$$
\begin{aligned}
\frac{d}{d t}\left[x^{\prime}(t)-\frac{1}{\lambda} g\left(t, x_{t}, \int_{0}^{t} a\left(t, s, x_{s}\right) d s\right)\right] & \in A x(t)+\frac{1}{\lambda} F\left(t, x_{t}, \int_{0}^{t} b\left(t, s, x_{s}\right) d s\right), t \in J=[0, T], \\
t \neq t_{k}, \quad k=1,2, \ldots, m & \\
x(t) & =\varphi \in \mathcal{B}_{h}, \quad x^{\prime}(0)=x_{1} \in E, \\
\left.\Delta x\right|_{t=t_{k}} & =\frac{1}{\lambda} I_{k}^{1}\left(x\left(t_{k}^{-}\right)\right), \quad k=1,2, \ldots, m, \\
\left.\Delta x^{\prime}\right|_{t=t_{k}} & =\frac{1}{\lambda} I_{k}^{2}\left(x\left(t_{k}^{-}\right)\right), \quad k=1,2, \ldots, m .
\end{aligned}
$$


Thus, by Definition 3.1, the mild solution of (3.4) can be written as

$$
\begin{aligned}
x(t)= & C(t) \varphi(0)+S(t)\left[x_{1}-g(0, \varphi, 0)\right]+\frac{1}{\lambda} \int_{0}^{t} C(t-s) g\left(s, x_{s}, \int_{0}^{s} a\left(s, \tau, x_{\tau}\right) d \tau\right) d s \\
& +\frac{1}{\lambda} \int_{0}^{t} S(t-s) f(s) d s+\sum_{0<t_{k}<t} C\left(t-t_{k}\right) I_{k}^{1}\left(x\left(t_{k}^{-}\right)\right) \\
& +\sum_{0<t_{k}<t} S\left(t-t_{k}\right) I_{k}^{2}\left(x\left(t_{k}^{-}\right)\right), \quad t \in J
\end{aligned}
$$

where

$$
f \in S_{F, x}=\left\{f \in L^{1}(J, E): f(t) \in F\left(t, x_{t}, \int_{0}^{t} b\left(t, s, x_{s}\right) d s\right) \text {, for a.e. } t \in J\right\} .
$$

Lemma 3.6. If hypotheses (H1)-(H6) are satisfied, let $x(t)$ be a mild solution of equation (3.4), then there exists a priori bound $\mathbb{K}>0$ such that $\left\|x_{t}\right\|_{\mathcal{B}_{h}} \leq \mathbb{K}, t \in J$, where $\mathbb{K}$ depends only on $T$ and on the functions $m(\cdot)$, $\Omega(\cdot)$ and $\Theta(\cdot)$.

Proof. From equation (3.5), we obtain

$$
\begin{aligned}
& |x(t)| \leq|C(t) \varphi(0)|+\left|S(t)\left[x_{1}-g(0, \varphi, 0)\right]\right|+\int_{0}^{t}\left|C(t-s) g\left(s, x_{s}, \int_{0}^{s} a\left(s, \tau, x_{\tau}\right) d \tau\right)\right| d s \\
& +\int_{0}^{t}|S(t-s) f(s)| d s+\sum_{0<t_{k}<t}\left|C\left(t-t_{k}\right) I_{k}^{1}\left(x\left(t_{k}^{-}\right)\right)\right| \\
& +\sum_{0<t_{k}<t}\left|S\left(t-t_{k}\right) I_{k}^{2}\left(x\left(t_{k}^{-}\right)\right)\right| \\
& \leq M_{0}|\varphi(0)|+M_{1}\left[\left|x_{1}\right|+L_{3}\|\varphi\|_{\mathcal{B}_{h}}+\widetilde{L}_{3}\right]+M_{0} \int_{0}^{t}\left[L_{3}\left[\left\|x_{s}\right\|_{\mathcal{B}_{h}}+\left\|\int_{0}^{s} a\left(s, \tau, x_{\tau}\right) d \tau\right\|\right]\right. \\
& \left.+\widetilde{L}_{3}\right] d s+M_{1} \int_{0}^{t} m(s) \Omega\left(\left\|x_{s}\right\|_{\mathcal{B}_{h}}+\left\|\int_{0}^{s} b\left(s, \tau, x_{\tau}\right) d \tau\right\|\right) d s+M_{0} \sum_{k=1}^{m} d_{k}+M_{1} \sum_{k=1}^{m} \tilde{d}_{k} \\
& \leq M_{0}|\varphi(0)|+M_{1}\left[\left|x_{1}\right|+L_{3}\|\varphi\|_{\mathcal{B}_{h}}+\widetilde{L}_{3}\right]+M_{0} T\left[L_{3} \widetilde{L}_{1}+\widetilde{L}_{3}\right] \\
& +M_{0} L_{3}\left[1+L_{1}\right] \int_{0}^{t}\left\|x_{s}\right\|_{\mathcal{B}_{h}} d s+M_{1} \int_{0}^{t} m(s) \Omega\left(\left\|x_{s}\right\|_{\mathcal{B}_{h}}+\int_{0}^{s} \gamma p(\tau) \Theta\left(\left\|x_{\tau}\right\|_{\mathcal{B}_{h}}\right) d \tau\right) d s \\
& +M_{0} \sum_{k=1}^{m} d_{k}+M_{1} \sum_{k=1}^{m} \tilde{d}_{k} \\
& \leq M_{0}\left[|\varphi(0)|+T\left(L_{3} \widetilde{L}_{1}+\widetilde{L}_{3}\right)+\sum_{k=1}^{m} d_{k}\right]+M_{1}\left[\left|x_{1}\right|+L_{3}\|\varphi\|_{\mathcal{B}_{h}}+\widetilde{L}_{3}+\sum_{k=1}^{m} \tilde{d}_{k}\right] \\
& +M_{0} L_{3}\left[1+L_{1}\right] \int_{0}^{t}\left\|x_{s}\right\|_{\mathcal{B}_{h}} d s+M_{1} \int_{0}^{t} m(s) \Omega\left(\left\|x_{s}\right\|_{\mathcal{B}_{h}}+\int_{0}^{s} \gamma p(\tau) \Theta\left(\left\|x_{\tau}\right\|_{\mathcal{B}_{h}}\right) d \tau\right) d s \\
& =K_{1}+M_{0} L_{3}\left[1+L_{1}\right] \int_{0}^{t}\left\|x_{s}\right\|_{\mathcal{B}_{h}} d s+M_{1} \int_{0}^{t} m(s) \Omega\left(\left\|x_{s}\right\|_{\mathcal{B}_{h}}+\int_{0}^{s} \gamma p(\tau) \Theta\left(\left\|x_{\tau}\right\|_{\mathcal{B}_{h}}\right) d \tau\right) d s .
\end{aligned}
$$

From Lemma 3.4, we get

$$
\begin{aligned}
\left\|x_{t}\right\|_{\mathcal{B}_{h}} \leq & \ell \sup \{|x(s)|: 0 \leq s \leq t\}+\|\varphi\|_{\mathcal{B}_{h}} \\
\leq & \|\varphi\|_{\mathcal{B}_{h}}+\ell K_{1}+\ell M_{0} L_{3}\left[1+L_{1}\right] \int_{0}^{t}\left\|x_{s}\right\|_{\mathcal{B}_{h}} d s \\
& +\ell M_{1} \int_{0}^{t} m(s) \Omega\left(\left\|x_{s}\right\|_{\mathcal{B}_{h}}+\int_{0}^{s} \gamma p(\tau) \Theta\left(\left\|x_{\tau}\right\|_{\mathcal{B}_{h}}\right) d \tau\right) d s, t \in J
\end{aligned}
$$

Let $u(t)=\sup \left\{\left\|x_{s}\right\|_{\mathcal{B}_{h}}: 0 \leq s \leq t\right\}$, then the function $u(t)$ is nondecreasing in $J$, and we have

$$
u(t) \leq h_{1}+h_{2} \int_{0}^{t} u(s) d s+h_{3} \int_{0}^{t} m(s) \Omega\left(u(s)+\int_{0}^{s} \gamma p(\tau) \Theta(u(\tau)) d \tau\right) d s, t \in J .
$$


Denoting by the right hand side of the above inequality as $v(t)$, we see that

$$
v(0)=h_{1}, \quad u(t) \leq v(t), t \in J
$$

and

$$
v^{\prime}(t)=h_{2} u(t)+h_{3} m(t) \Omega\left(u(t)+\int_{0}^{t} \gamma p(s) \Theta(u(s)) d s\right) .
$$

Since $\Omega$ is nondecresing

$$
v^{\prime}(t) \leq h_{2} v(t)+h_{3} m(t) \Omega\left(v(t)+\int_{0}^{t} \gamma p(s) \Theta(v(s)) d s\right), t \in J .
$$

Let

$$
w(t)=v(t)+\int_{0}^{t} \gamma p(s) \Theta(v(s)) d s
$$

Then

$$
\begin{aligned}
& w(0)=v(0) \quad \text { and } \quad v(t) \leq w(t) . \\
w^{\prime}(t)= & v^{\prime}(t)+\gamma p(t) \Theta(v(t)) \\
\leq & h_{2} v(t)+h_{3} m(t) \Omega(w(t))+\gamma p(t) \Theta(v(t)) \\
\leq & h_{2} w(t)+h_{3} m(t) \Omega(w(t))+\gamma p(t) \Theta(w(t)) \\
\leq & \widetilde{m}(t)[w(t)+\Omega(w(t))+\Theta(w(t))] .
\end{aligned}
$$

This implies

$$
\int_{w(0)}^{w(t)} \frac{d s}{s+\Omega(s)+\Theta(s)} \leq \int_{0}^{T} \widetilde{m}(s) d s<\int_{h_{1}}^{\infty} \frac{d s}{s+\Omega(s)+\Theta(s)}, \quad t \in J .
$$

This inequality implies that $w(t)<\infty$. Hence there is a constant $\mathbb{K}$ such that $w(t) \leq \mathbb{K}$, $t \in J$. Thus, we have $\left\|x_{t}\right\|_{\mathcal{B}_{h}} \leq u(t) \leq v(t) \leq w(t) \leq \mathbb{K}, t \in J$, where $\mathbb{K}$ depends only on $T$ and on the functions $m(\cdot), \Omega(\cdot)$ and $\Theta(\cdot)$.

Theorem 3.1. Assume that the hypotheses (H1)-(H6) hold. Then the problem (1.1)-(1.4) admits at least one solution on $J$.

Proof. Let $G=\left\{y \in \mathcal{B}_{h}^{\prime \prime}: \lambda y \in \Phi_{1} y \quad\right.$ for some $\left.\lambda \in(0,1)\right\}$. Then for any $y \in G$, we have

$$
\begin{aligned}
y(t)= & \frac{1}{\lambda} S(t)\left[y_{1}-g(0, \varphi, 0)\right]+\frac{1}{\lambda} \int_{0}^{t} C(t-s) g\left(s, y_{s}+\tilde{\varphi}_{s}, \int_{0}^{s} a\left(s, \tau, y_{\tau}+\tilde{\varphi}_{\tau}\right) d \tau\right) d s \\
& +\frac{1}{\lambda} \int_{0}^{t} S(t-s) f(s) d s+\frac{1}{\lambda} \sum_{0<t_{k}<t} C\left(t-t_{k}\right) I_{k}^{1}\left(y\left(t_{k}^{-}\right)+\tilde{\varphi}\left(t_{k}^{-}\right)\right) \\
& +\frac{1}{\lambda} \sum_{0<t_{k}<t} S\left(t-t_{k}\right) I_{k}^{2}\left(y\left(t_{k}^{-}\right)+\tilde{\varphi}\left(t_{k}^{-}\right)\right)
\end{aligned}
$$

which implies the function $x=y+\tilde{\phi}$ is a mild solution of above system (3.4), for which we have proved in Lemma 3.6 as $\left\|x_{t}\right\|_{\mathcal{B}_{h}} \leq \mathbb{K}, t \in J$, and hence from Lemma 3.4

$$
\begin{aligned}
\|y\|_{T} & =\left\|y_{0}\right\|_{\mathcal{B}_{h}}+\sup \{|y(t)|: 0 \leq t \leq T\} \\
& =\sup \{|y(t)|: 0 \leq t \leq T\} \\
& \leq \sup \{|x(t)|: 0 \leq t \leq T\}+\sup \{|\tilde{\varphi}(t)|: 0 \leq t \leq T\} \\
& \leq \sup \left\{l^{-1}\left\|x_{t}\right\|_{\mathcal{B}_{h}}: 0 \leq t \leq T\right\}+\sup \{|C(t) \varphi(0)|: 0 \leq t \leq T\} \\
& \leq l^{-1} \mathbb{K}+M_{0}|\varphi(0)|
\end{aligned}
$$

which implies that the set $G$ is bounded on $J$.

Hence it follows from Lemma 3.5 and Theorem 2.1 that the operator $\Phi_{1}$ has a fixed point $y^{*} \in \mathcal{B}_{h}^{\prime \prime}$. Let $x(t)=$ $y^{*}(t)+\tilde{\varphi}(t), t \in(-\infty, T]$. Then $x$ is a fixed point of the operator $\Phi$ which is a mild solution of the problem (1.1)-(1.4). 


\section{Acknowledgements:}

The authors dedicate this paper to "Silver Jubilee Year Celebrations of Karunya University, Coimbatore-641 114, Tamil Nadu, India". And also the authors wish to thank Dr. Paul Dhinakaran, Chancellor, Dr. Paul P. Appasamy, ViceChancellor, and Dr(Mrs). Anne Mary Fernandez, Registrar, of Karunya University, Coimbatore, for their constant encouragements and support for this research work.

\section{References}

[1] M. Adimy and K. Ezzinbi, Strict solutions of nonlinear hyperbolic neutral differential equations, Appl. Math. Lett., 12:1 (1999) 107-112. 1

[2] A. Anguraj and M. Mallika Arjunan, Existence and uniqueness of mild and classical solutions of impulsive evolution equations, Electronic Journal of Differential Equations, 2005(111)(2005), 1-8. 1

[3] D.D. Bainov and P.S. Simeonov, Impulsive Differential Equations: Periodic Solutions and Applications, Longman Scientific and Technical Group, England, 1993. 1

[4] K. Balachandran, J.Y. Park and S.H. Park, Controllability of nonlocal impulsive quasi-linear integrodifferential systems in Banach spaces, Reports on Mathematical Physics, 65(2)(2010), 247-257. 1

[5] K. Balachandran and N. Annapoorani, Existence results for impulsive neutral evolution integrodifferential equations with infinite delay, Nonlinear Analysis: Hybrid Systems, 3 (2009), 674-684. 1

[6] J. Banas and K. Goebal, Measures of Noncompactness in Banach spaces, Dekker, New York, 1980.2

[7] M. Benchohra, J. Henderson and S.K. Ntouyas, Impulsive Differential Equations and Inclusions, Hindawi Publishing Corporation, 2006. 1

[8] M. Benchohra, J. Henderson and S.K. Ntouyas, An existence result for first order impulsive functional differential equations in Banach spaces, Comp. Math. Appl., 42 (2001), 1303-1310. 1

[9] M. Benchohra, J. Henderson and S.K. Ntouyas, Semilinear impulsive neutral functional differential inclusions in Banach spaces, Appl. Anal., 81:4 (2002), 951-963. 1

[10] M. Benchohra and A. Ouahab, Impulsive neutral functional differential equations with variable times, Nonlinear Analysis, $\mathbf{5 5 : 6}$ (2003), 679-693. 1

[11] M. Benchohra and S.K. Ntouyas, Existence and controllability results for nonlinear differential inclusions with nonlocal conditions, J. Appl. Anal., 8 (2002), 31-46. 1

[12] M. Benchohra and A. Ouahab, Impulsive neutral functional differential inclusions with variable times, Electronic Journal of Differential Equations, 2003:67 (2003), 1-12. 1

[13] M. Benchohra, E.P. Gatsori, J. Henderson, and S.K. Ntouyas, Nondensely defined evolution impulsive differential inclusions with nonlocal conditions, J. Math. Anal. Appl., 286 (2003), 307-325. 1

[14] M. Benchohra, J. Henderson and S.K. Ntouyas, Existence results for impulsive multivalued semilinear neutral functional differential inclusions in Banach Spaces, J. Math. Anal. Appl., 263(2001), 763-780. 1

[15] Y.K. Chang, A. Anguraj and M. Mallika Arjunan, Existence results for impulsive neutral functional differential equations with infinite delay, Nonlinear Analysis: Hybrid Systems, 2(1)(2008), 209-218. 1 [2]

[16] Y. -K. Chang and W.-T. Li, Existence results for second order impulsive functional differential inclusions, J. Math. Anal. Appl., 301:2 (2005), 477-490. 1

[17] Y. -K. Chang and Li. Mei. Qi, Existence results for second order impulsive functional differential inclusions, J. Appl. Math. Stochastic Anal., 2006, 1-12. 1 .

[18] Y. -K. Chang, Controllability of impulsive functional differential systems with infinite delay in Banach spaces, Chaos, Solitons \& Fractals, 33 (2007), 1601-1609. 1 [1.4

[19] Y.-K. Chang, M. Mallika Arjunan and V. Kavitha, Existence results for a second order impulsive functional differential equations with state-dependent delay, Differential Equations and Applications, 1:3(2009), 325-339. 1]

[20] Y. -K. Chang, A. Anguraj and K. Karthikeyan, Existence for impulsive neutral integrodifferential inclusions with nonlocal initial conditions via fractional operators, Nonlinear Anlaysis, 71(2009), 4377-4386. 1 .

[21] K. Deimling, Multivalued Differential Equations, Walter de Gruyter, Berlin, New York, 1992.2

[22] L. Erbe and W. Krawcewicz, Existence of solution to B.V.P for impulsive second order differential inclusions, Rocky Mountain J. Math. 22:2 (1992), 519-539. 1

[23] M. Frigon and D. O' Regan, Boundary value problems for second order impulsive differential equations using set-valued maps, Appl. Anal., 58:3-4(1995), 325-333. 1

[24] X. Fu, Y. Cao, Existence for neutral impulsive differential inclusions with nonlocal conditions, Nonlinear Analysis: Theory, Methods and Applications, 68:12(2008), 3707-3718. 1

[25] J. K. Hale and J. Kato, Phase space for retarded equations with infinite delay, Funckcial. Ekvac., 21 (1978), 11-41. 1

[26] J. K. Hale, L. Verduyn and M. Sjoerd, Introduction to functional differential equations, Appl. Math. Sci., 99. Springer Verlag, New York, 1993. 1

[27] J. K. Hale, Partial neutral functional differential equations, (English. English summary) Rev. Roumaine Math. Pures Appl., 39:4 (1994), 339-344. 1

[28] E. Hernandez, A second order impulsive Cauchy problem, Int. J. Math. Math. Sci., 31(8)(2002), 451-461. 1

[29] E. Hernandez and H.R. Henriquez, Impulsive partial neutral differential equations, Appl. Math. Lett., 19(2006), 215-222. 1

[30] E. Hernández, H.R. Henríquez and R. Marco, Existence of solutions for a class of impulsive partial neutral functional differential equations, J. Math. Anal. Appl., 331:2 (2007), 1135-1158. 1

[31] E. Hernández, Existence results for a partial second order functional differential equation with impulses, Dynamics of Continuous, Discrete and Impulsive Systems, Series A: Mathematical Analysis, 14 (2007) 229-250. 1

[32] E. Hernández, H.R. Henríquez, M.A. Mckibben, Existence results for abstract impulsive second-order neutral functional differential equations, Nonlinear Analysis:Theory, Methods and Applications, 70 (2009) 2736-2751. 1

[33] E. Hernández, Marco Rabello, Hernn R. Henríquez, Existence of solutions for impulsive partial neutral functional differential equations, J. Math. Anal. Appl., 331:2(2007), 1135-1158. 1 
[34] E. Hernández and H.R. Henríquez, Existence results for partial neutral functional differential equations with unbounded delay, J. Math. Anal. Appl., 221:2 (1998), 452-475. 1

[35] E. Hernández and H.R. Henríquez, Existence of periodic solutions of partial neutral functional differential equations with unbounded delay, J. Math. Anal. Appl., 221:2 (1998), 499-522. 1 .

[36] E. Hernández, Existence results for partial neutral integrodifferential equations with unbounded delay, J. Math. Anal. Appl., 292:1(2004), 194-210. 1

[37] Y. Hino, S. Murakami and T. Naito, Functional Differential Equations with Infinite Delay, in: Lecture Notes in Mathematics, Vol.1473, Springer-Verlag, Berlin, 1991. 1]

[38] Sh. Hu and N. Papageorgiou, Handbook of Multivalued Analysis, Volume 1: Theory, Kluwer, Dordrecht, Boston, London, 1997. 2

[39] J. Hu and X. Liu, Existence results of second order impulsive neutral functional integrodifferential inclusions with unbounded delay in Banach spaces, Mathematical and Computer Modelling, 49(2009), 516-526. 1 .

[40] Kisyński, J., On cosine operator functions and one parameter group of operators, Studia Math., 49(1972), 93-105. 2

[41] V. Kolmanovskii, A. Myshkis, Applied Theory of Functional Differential Equations, Kluwer, Dordrecht, 1992. 1

[42] V. Kolmanovskii, A. Myshkis, Introduction to the Theory and Applications of Functional Differential Equations, Kluwer, Dordrecht, 1999. 1

[43] V. Lakshmikantham, D.D. Bainov and P.S. Simeonov, Theory of Impulsive Differential Equations, World Scientific, NJ, 1989. 1

[44] A. Lasota and Z. Opial, An application of the Kakutani-Ky Fan theorem in the theory of ordinary differential equations, Bull. Acad. Pol. Sci. Ser. Sci. Math. Astronom. Phys., 13 (1965), 781-786. 3.2] 3.3

[45] J. Liang, J.H. Liu and T.-J. Xiao, Nonlocal impulsive problems for nonlinear differential equations in Banach spaces, Math. Comput. Model., 49(3-4)(2009), 794-804. 1

[46] B. Liu, Controllability of impulsive neutral functional differential inclusions with infinite delay, Nonlinear Analysis, 60(2005), 1533-1552.

[47] J.H. Liu, Nonlinear impulsive evolution equations, Dyn. Contin. Discrete Impuls. Syst., 6(1)(1999), 77-85. 1

[48] M. Martelli, A Rothe's type theorem for non-compact acyclic-valued map, Boll. Un. Mat. Ital., 11(1975), 70-76 2.1

[49] S.K. Ntouyas, Existence results for impulsive partial neutral functional differential inclusions, Electronic J. Differential Equations, 2005:30 (2005), 1-11. 1

[50] J.Y. Park, K. Balachandran and N. Annapoorani, Existence results for impulsive neutral functional integrodifferential equations with infinite delay, Nonlinear Analysis, 71(2009), 3152-3162. 1

[51] A.M. Samoilenko and N.A Perestyuk, Impulsive Differential Equations, World Scientific, Singapore, 1995. 1

[52] Travis, C. C. and Webb, G. F., Compactness, regularity, and uniform continuity properties of strongly continuous cosine families, Houston J. Math., 3 (4)(1977) 555-567. 2 2

[53] Travis, C. C. and Webb, G. F., Cosine families and abstract nonlinear second order differential equations, Acta Math.Acad. Sci. Hungaricae, 32(1978), 76-96. 2 2

[54] Y.V. Rogovchenko, Nonlinear impulsive evolution systems and applications to population models, J. Math. Anal. Appl., 207(2)(1997), 300-315. 1

[55] J. Wu and H. Xia, Self-sustained oscillations in a ring array of coupled lossless transmission lines, J. Differential Equations, 124:1 (1996), 247-278. 1

[56] J. Wu, Theory and Applications of Partial Functional Differential Equations, Appl. Math. Sci. 119. Springer Verlag, New York, 1996. 1

[57] B. Yan, Boundary value problems on the half-line with impulses and infinite delay, J. Math. Anal. Appl., 259 (2001), 94-114. 1 